\section{Perfil de beta talassemia heterozigota obtido a partir de análise data mining em banco de dados}

\section{The profile of beta thalassemia obtained by data mining analysis in a database}

\author{
Ana L. B. Domingos ${ }^{1}$ \\ Lucas A. Granzotto ${ }^{2}$ \\ Edis Belini Junior ${ }^{3}$ \\ Thiago Y. K. Oliveira ${ }^{3}$ \\ Ana C. B. Domingos ${ }^{4}$ \\ Claudia R. Bonini-Domingos ${ }^{5}$ \\ ${ }^{1}$ Iniciação Científica - Depto. de Química, Unesp, São José do Rio \\ Preto-SP \\ ${ }^{2}$ Iniciação Cientifica - Depto. de Ciências da Computação, Unesp, \\ São José do Rio Preto-SP \\ ${ }^{3}$ Biólogo, Hemocentro USP - Ribeirão Preto-SP \\ ${ }^{4}$ Farmacêutica bioquímica. Programa de Pós-Graduação em \\ Genética, Unesp, São José do Rio Preto-SP \\ ${ }^{5}$ Professora. Depto. de Biologia, Unesp, São José do Rio Preto-SP
}

\section{Sr. Editor}

As doenças causadas por hemoglobinas variantes são responsáveis por cerca de 3,4\% das mortes ocorridas antes dos cinco anos de idade, ${ }^{1,2,3}$ e no Brasil, em especial, com as migrações e miscigenação da população, uma gama de hemoglobinas está presente. Por esse motivo torna-se necessário a associação de análise laboratorial clássica e molecular, a fim de se desenvolverem perfis para os casos mais graves e facilitar a criação de uma política de saúde específica para as regiões em que ocorrem com maior frequência. ${ }^{3,4,5} \mathrm{~A}$ beta talassemia na forma heterozigota é caracterizada pela elevação dos valores de $\mathrm{Hb} \mathrm{\textrm {A } _ { 2 }}$ e/ou Hb Fetal nos casos clássicos. $., 6,7$ $\mathrm{O}$ acréscimo dos valores da $\mathrm{Hb} \mathrm{A}_{2}$ pode sugerir o genótipo do indivíduo. Variações na expressão fenotípica são observadas, refletindo a formação da nossa população. ${ }^{6-9}$ Para se conhecer o perfil da beta talassemia heterozigota na população brasileira objetivou-se estabelecer parâmetros de norteamento de diagnóstico e a frequência de portadores a partir das informações depositadas em um banco de dados.

Foram avaliadas as informações referentes a 10.960 laudos de diagnóstico, depositados em uma base de dados, entre o período de janeiro de 2002 e julho de 2008. Os resultados de testes laboratoriais foram incluídos em banco de dados em modelo relacional e na linguagem SQL. As informações foram analisadas pela ferramenta data mining CLIBIA (Clinical Bioinformatic Analysis). ${ }^{10,11,12}$ Formularamse as seguintes faixas de corte e classificação dos grupos, para estabelecer os perfis de beta talassemia heterozigota, segundo os padrões estabelecidos para as condições laboratoriais e população em estudo:

- $\mathrm{Hb} \mathrm{A}_{2}$ aumentada: com valores acima de 3,99\%;

- $\mathrm{Hb} \mathrm{A}_{2}$ discretamente aumentada: com valores entre $3,51 \%$ até $3,98 \%$;

- $\mathrm{Hb} \mathrm{A}_{2}$ e $\mathrm{Hb} \mathrm{F}$ aumentadas: $\mathrm{Hb} \mathrm{A}_{2}$ acima de 3,99\% e Hb F acima de $1,51 \%$;

- $\mathrm{Hb} \mathrm{A}_{2}$ e $\mathrm{Hb} \mathrm{F}$ discretamente aumentadas: $\mathrm{Hb} \mathrm{A}_{2}$ entre 3,51\% até 3,98\% e $\mathrm{Hb} \mathrm{F}$ entre 1,01\% até 1,5\%.

Do total de amostras avaliadas, provenientes de todas as regiões do País, 868 (7,92\%) apresentaram valores dentro dos perfis inicialmente estabelecidos, como critérios de corte. A frequência observada para cada região, no período de sete anos foi: 3,50\% para a região Sul; 21,05\% para a região Centrooeste; $3,95 \%$ para o Nordeste; $1,20 \%$ para o Norte e $8,66 \%$ para a região Sudeste. Não houve diferença significativa entre os sexos. Os valores encontrados para a região centro-oeste refletem a dificuldade diagnóstica enfrentada pelos serviços de diagnóstico e a necessidade da busca por serviços de referência no auxilio diagnóstico.

A avaliação refinada pelo instrumento de prospecção mostrou que, no ano de 2002, início dessa pesquisa, o número de casos avaliados foi insuficiente para uma análise global. Em 2003, destacou-se o perfil de $\mathrm{Hb} \mathrm{\textrm {A } _ { 2 }}$ aumentada + $\mathrm{Hb} \mathrm{F}$ como o mais frequente, conforme mostra a Figura 1. Em 2004 houve um aumento do perfil caracterizado como $\mathrm{Hb} \mathrm{A}_{2}$ aumentada, que prevaleceu sobre os outros e se manteve constante até o período atual, resultado evidenciado na Figura 2.

Os perfis mais brandos foram pouco significativos na análise global, sendo o perfil de $\mathrm{Hb} \mathrm{A}_{2}$ discretamente aumentada,o mais evidente a partir de 2005.

$\mathrm{O}$ maior número de registros de suspeitas de beta talassemia ocorreu em 2006 e representaram cerca de 15,70\% do total de amostras avaliadas, correspondendo a 474 casos. Neste mesmo ano, houve um acréscimo na identificação de casos com o perfil de $\mathrm{Hb} \mathrm{A}_{2}$ aumentada $+\mathrm{Hb} \mathrm{F}$ e do perfil de $\mathrm{Hb} \mathrm{A}_{2}$ discretamente aumentada, decorrentes principalmente da implantação de metodologias mais precisas e para a quan-

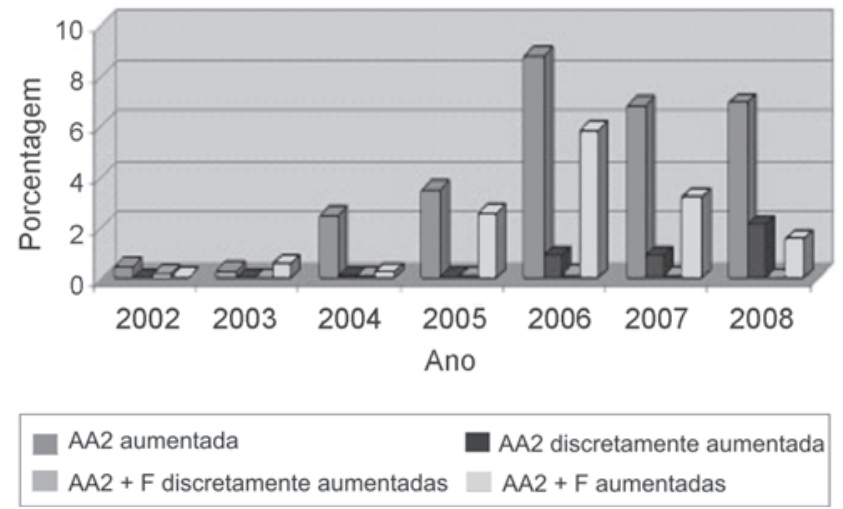

Figura 1. Gráfico da variação dos perfis estabelecidos de beta talassemia em relação ao número total de amostras enviadas por ano 


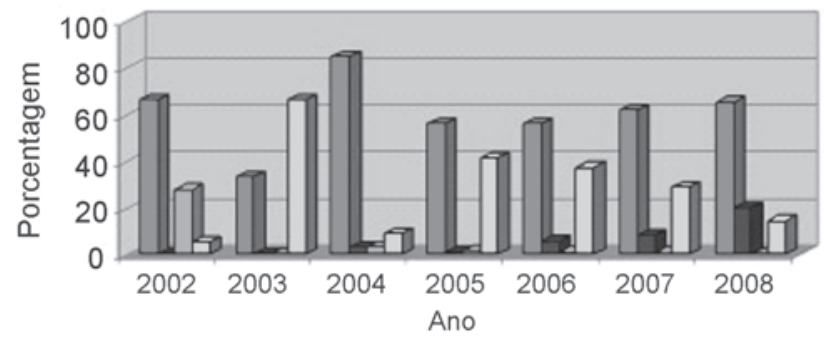

AA2 aumentada

$\mathrm{AA} 2+\mathrm{F}$ discretamente aumentadas

AA2 discretamente aumentada $\mathrm{AA} 2+\mathrm{F}$ aumentadas

Figura 2: Gráfico da variação dos perfis estabelecidos de beta talassemia em relação ao número total de suspeitas enviadas por ano

tificação das frações de hemoglobinas, como a HPLC. Ressaltamos que as suspeitas laboratoriais, obtidas por metodologias clássicas, só podem ser confirmadas por meio de análise molecular. Nesse estudo evidenciamos que $60 \%$ das amostras com perfis de $\mathrm{Hb} \mathrm{\textrm {A } _ { 2 }}$ aumentada e $\mathrm{Hb} \mathrm{A}_{2}$ aumentada $+\mathrm{Hb} \mathrm{F}$ foram de casos de beta zero talassemia, principalmente da mutação CD 39.

Assim, a análise dos perfis de beta talassêmicos heterozigotos, obtido por meio de instrumento Data Mining, mostrou que a grande maioria dos casos representou um perfil de beta zero talassemia, com aumento de $\mathrm{Hb} \mathrm{\textrm {A } _ { 2 }}$ acima de 3,99\%, refletindo a formação da população brasileira, principalmente da região Sudeste. O aumento na solicitação dos exames e a melhoria e precisão das metodologias de análise destacaram a importância do diagnóstico para essa forma hereditária de anemia e refletiram a preocupação e a importância que os profissionais de saúde apresentam em propor melhorias no diagnóstico laboratorial.

\begin{abstract}
Variations in the phenotypic expression of heterozygous beta thalassemia reflect the formation of different populations. To better understand the profile of heterozygous beta-thalassemia of the Brazilian population, we aimed at establishing parameters to direct the diagnosis of carriers and calculate the frequency from information stored in an electronic database. Using a Data Mining tool, we evaluated information on 10,960 blood samples deposited in a relational database. Over the years, improved diagnostic technology has facilitated the elucidation of suspected beta thalassemia heterozygote cases with an average frequency of 3.5\% of referred cases. We also found that the Brazilian beta thalassemia trait has classic increases of $\mathrm{Hb} \mathrm{A} 2$ and $\mathrm{Hb} F(60 \%)$, mainly caused by mutations in beta zero thalassemia, especially in the southeast of the country. Rev. Bras. Hematol. Hemoter. 2009;32(1):78-79.
\end{abstract}

Key words: Data mining; beta thalassemia trait; bioinformatics; genetic diversity

\section{Referências Bibliográficas}

1. Honig GR, Adams III JG. Human hemoglobin genetics, wien, N.Y., Springer Verlag, 1986.

2. Zago MA. Hemoglobinopathies: recent advances and collaborative research. Ciência e Cultura. 1987;39:829-33.

3. Cançado RD, Jesus JA. A doença falciforme no Brasil. Rev. Bras. Hematol. Hemoter. 2007;29(3):203-6.

4. Bonini-Domingos CR. Metodologias laboratoriais para o diagnóstico de hemoglobinopatias e talassemias. Ed. HN, São José do Rio Preto, 2006

5. Weatherall DJ, Clegg JB. The thalassemia syndromes. $3^{\text {rd. }}$ edition/ Blackwell scientific Publications.1981

6. Weatherall DJ. The thalassemias. Brit Med J. 1997;314:1675-8.

7. Hardison RS, Chui DHK, Giardine B, et al. HbVar: A relational database of human hemoglobin variants and thalassemia mutations at the globin gene server. Human Mutation. 2002. http:// globin.cse.psu.edu/hbvar/menu.html acesso em setembro de 2009.

8. Huisman HJ, Marianne FH, Carver EB, Efremov GD. Hb Var: a database of human hemoglobin variants and thalassemias, summaries of mutation categories. Pennsylvania University USA and McMaster University in Canada, 2001. Disponível em <http:// globin.cse.psu.edu/>. Acesso em setembro de 2009.

9. Hünemeier T, Carvalho C, Marrero RA, et al. Niger-Congo speaking populations and the formation of the Brazilian gene pool: mtDNA and Y-chromosome data. Am J Phys Anthropol. 2007;133(2):85467.

10. Elmasri R. Fundamentals of Database Systems, $3^{\text {a }}$ Editions, ISBN 0-201-54263-3, Addison-Wesley, 2000

11. Korth S, Sudarshan. Sistema de Banco de Dados, $3^{a}$ Edição, 1999.

12. Korth HF. \& Silberschatz A. Sistema de Bancos de Dados. São Paulo, MacGraw-Hill, 1989.

Avaliação: Editor e dois revisores externos

Conflito de interesse: sem conflito de interesse

Recebido: 03/07/2009

Aceito após modificações: 24/09/2009

Unesp, Departamento de Biologia, Laboratório de Hemoglobinas e Genética das Doenças Hematológicas - LHGDH, Unesp/Ibilce

Correspondência: Claudia Regina Bonini-Domingos Laboratório de Hemoglobinas e Genética das Doenças Hematológicas

Rua Cristóvão Colombo, 2265 - Jd. Nazareth 15054-000 - São José do Rio Preto-SP - Brasil Tel.: (55 17) 3221-2392; Fax: (55 17)3221-2390 E-mail: claudiabonini@sjrp.unesp.br 